\title{
IDIOMATIC EXPRESSIONS AND TEACHING OF FRENCH AS A FOREIGN LANGUAGE: PROBLEM AND PERSPECTIVES
}

\author{
[EXPRESSIONS IDIOMATIQUES ET L'ENSEIGNEMENT DU \\ FRANÇAIS LANGUE ETRANGERE: PROBLEMATIQUE ET \\ PERSPECTIVES]
}

\author{
Sonja Spadijer - Carey Dardompre
}

DOI: 10.18355/PG.2015.4.1.89-107

\begin{abstract}
Idiomatic expressions, defined as polylexical fixed expressions with a figurative sense, make up an important segment of the French lexicon which should have a larger role in methods and curricula for teaching French as a foreign language (FFL). A few examples are: se faire les dents, avoir la dent, faire les yeux en coulisses, avoir l'œil américain. Given that the study of idiomatic expressions is also an intercultural matter, we find that it is one of the most important topics in an FFL classroom. We hope that this article will draw the attention of teachers, authors, and publishing houses to the importance of a more coherent approach in the study of French idiomatic expressions. We summarize the current situation concerning the place of idiomatic expressions in the official curricula and textbooks of FFL in Montenegro and in Massachusetts. In addition, we propose strategies and modalities so that idiomatic expressions are integrated systematically into the pedagogy of FFL. The present study rests on the analysis of the official curricula and pedagogical methods of both Montenegro and Massachusetts, and on the results of a semic analysis aiming at definitional paraphrases of idiomatic expressions. We noticed some inconsistency concerning the importance of idiomatic expressions in certain curricula and in a large number of textbooks. The semic analysis of the lexicographical definitions allows us to propose varied content with which to organize the study of idiomatic expressions in FFL classrooms using a greater variety of classroom techniques than currently exist.
\end{abstract}

Key words

idiomatic expressions, interculturalism, pedagogy, definitional circumlocution, empowering students

\section{Résumé}

Les expressions idiomatiques présentent un segment important du lexique français qui devrait trouver plus de place dans les programmes scolaires et les méthodes de français langue étrangère. Leurs caractéristiques principales sont le sens figuré, le figement et la polylexicalité. En voici quelque exemples : se faire les dents, avoir la dent, faire les yeux en coulisses, avoir l'œil américain. Nous sommes persuadés que les résultats de nombreuses recherches en ce domaine devraient trouver leur place dans la conception des programmes et méthodes de français langue étrangère. Notre objectif est de faire un état des 
lieux concernant leur place dans les programmes scolaires officiels ainsi que dans les manuels de français langue étrangère au Monténégro et au Massachusetts. Outre cela, nous avons l'intention de proposer des stratégies et des modalités pour que les expressions idiomatiques soient intégrées de façon plus systématique dans l'enseignement de français. La présente recherche repose d'une part sur l'analyse des programmes scolaires officiels et des méthodes de français en usage dans les deux Etats mentionnés cidessus et, d'autre part, sur les résultats d'une analyse sémique visant les périphrases définitionnelles des expressions idiomatiques. Nous avons constaté l'inconsistance concernant l'importance des expressions idiomatiques pour l'apprentissage de français langue étrangère dans certains programmes scolaires et chez une grande partie d'auteurs de méthodes de français. L'analyse sémique des définitions lexicographiques nous a permis de proposer des contenus variés à partir desquels il serait possible d'organiser le travail sur les expressions idiomatiques dans la classe de français langue étrangère. Ce travail se caractériserait d'une plus grande variété en matière de techniques de classe par rapport à celles existant jusqu'à présent. Vu que l'apprentissage des expressions idiomatiques relève aussi de l'interculturel, nous trouvons que c'est l'un des sujets le plus important dans la classe de français langue étrangère. Nous espérons que cet article attirera l'attention des responsables de la politique d'enseignement de français langue étrangère, enseignants, auteurs et maisons d'édition, sur l'importance d'une approche plus cohérente dans l'apprentissage des expressions idiomatiques.

\section{Mots clés}

les expressions idiomatiques, l'interculturel, la didactique, la périphrase définitionnelle, capable de parole.

\section{Introduction}

De nos jours, les expressions idiomatiques présentent un sujet d'actualité non seulement en français, mais dans un grand nombre de langues. En matière du lexique français, c'est un segment qui, par sa diversité morphosyntaxique et sémantique, inspire de nombreuses recherches qui essaient d'établir et de définir la terminologie à adopter, les critères de leur classification, la méthodologie à appliquer dans les analyses, le rapport entre le sens littéral et le sens figuré, le degré et la portée de figement, la manière de présentation et de traitement dans les dictionnaires ainsi que leur rôle dans le discours.

Nous considérons que ce grand intérêt pour les expressions idiomatiques observées dans le cadre de la linguistique devrait se répercuter également sur le domaine de la didactique de français langue étrangère et sur les politiques de l'apprentissage de français.

La question que nous aimerions soulever dans cet article est la suivante :

Les expressions idiomatiques, ont-elles une place méritée dans les programmes scolaires de français langue étrangère et dans les méthodes de français? D'abord, nous ferons le point sur la situation actuelle au Monténégro. L'enjeu une fois établi, nous tenterons de faire un 
rapprochement entre le programme scolaire de l'état de Massachusetts et celui de Monténégro. Notre objectif sera d'exposer les cinq composantes du programme scolaire langue étrangère (Massachusetts Foreign Languages Curriculum Framework, August 1999), puis d'essayer de démontrer comment cet outil communément interpelé «les $5 \mathrm{c}{ }^{1}$ permet l'intégration des expressions idiomatiques dans la didactique des langues.

Tout d'abord, nous citerons quelques caractéristiques majeures des expressions idiomatiques françaises.

Les expressions idiomatiques constituent un segment important du lexique français. En français, leur présence est considérable (Mejri, 2003: 24) et elles sont en usage dans tous les registres de langue.

Nous nous référons ici aux unités polylexicales qui dans le discours se présentent comme un ensemble qui a sa propre signification. D'habitude, elles ont un sens plus ou moins transparent ou bien complètement opaque. Malgré leur apparence qui peut évoquer la forme du syntagme libre et le fait qu'elles fonctionnent d'après les règles syntagmatiques, ce sont des suites figées qui ont les possibilités limitées de variabilité. L'ordre de mots peut varier et parfois, certains mots peuvent être remplacés par d'autres sans que cela entraîne un changement au niveau de l'image ou de la métaphore.

En français, ces expressions figurent sous le nom de locutions verbales ou expressions verbales idiomatiques (Gross, 1996 : 69). Elles sont issues du processus de figement lexical : «[...] processus de stabilisation du signifiant et du signifié d'une séquence polylexicale, aboutissant au codage dans le lexique » (Mortureux, 2008: 206).

Leur signification est arbitraire et conventionnelle. Les expressions verbales idiomatiques sont les phrases conventionnelles dont le sens diffère par rapport aux sens de leurs composantes (Knowles - Moon, 2006 : 19).

Dans cet article, nous faisons référence aux expressions constituées à priori d'un lexème désignant la tête et le corps humain. En voici quelques exemples: se faire les dents, rouler sa bosse, souffler dans des vessies trouées, avoir mal aux cheveux, couper/fendre les cheveux en quatre, ne pas avoir de plomb dans la tête, vouloir prendre la lune avec les dents, être la tête d'affiche, avoir le dos souple, faire grise mine, avoir la dent, avoir l'estomac dans les talons, casser les pieds à quelqu'un, se mettre les doigts dans l'œil, avoir les coudées franches.

Notre recherche vise donc des structures complexes, c'est-à-dire des syntagmes et des phrases, dont les caractéristiques principales sont : le figement, la polylexicalité, le sens figuré et la non-transparence sémantique.

Précisons tout de même que le nombre des expressions complètement opaques en français n'est pas trop élevé, comme l'affirme G.

\footnotetext{
${ }^{1}$ Les composantes de ce curriculum sont au nombre de cinq: Communication, Cultures, Comparaisons, Connections, et Communautés (Communication, Cultures, Comparisons, Connections, and Communities).
} 
Gross en se référant aux recherches effectuées dans le cadre de LADL et LLI ${ }^{1}$ (1996: 16, 17, 22).

Le fond lexical d'une langue fait partie du patrimoine culturel qu'il faut mettre en valeur et engager dans l'apprentissage d'une langue. Ainsi, les expressions idiomatiques reflètent les réalités sociales et historiques dans lesquelles cette langue existe. Pour les apprenants de français langue étrangère, connaître les expressions idiomatiques françaises dont la caractéristique principale est le sens figuré veut dire acquérir une bonne maîtrise de langue mais aussi les connaissances socioculturelles liées à l'usage de cette langue et à ses utilisateurs. Étant donné que les expressions idiomatiques présentent un phénomène lexical complexe caractérisé par le figement et la non-transparence sémantique, il est important de connaître leurs valeurs stylistiques, plus particulièrement les tournures comiques et autres effets stylistiquement marqués qui pourraient se produire suite à une rupture inattendue de figement, à l'introduction d'autres constituantes lexicales ou bien à l'interprétation littérale voulue de leur sens.

Outre cela, nous essaierons de mettre en avant d'autres raisons qui justifient l'apprentissage des expressions idiomatiques et la nécessité de leur présence dans les programmes scolaires, les objectifs, les compétences et savoir-faire concernant la didactique de français langue étrangère.

\section{L'importance des expressions idiomatiques dans la classe de français langue étrangère}

L'étude portant sur les expressions idiomatiques exige une approche interdisciplinaire vu qu'il s'agit d'un phénomène linguistique complexe. C'est pourquoi il faut permettre aux apprenants de différents niveaux de s'approprier de la richesse lexicale, de la valeur sémantique, de l'originalité, des caractéristiques stylistiques et des enjeux culturels des expressions idiomatiques.

Premièrement, nous allons souligner les enjeux culturels de l'apprentissage des expressions idiomatiques pour les apprenants. La façon indirecte de dire des choses grâce au sens figuré observé dans le sémantisme de ces expressions est très importante pour l'acquisition des stratégies communicatives et des façons de faire et de dire propre à la langue française. Il est important de prêter attention à l'importance de l'emploi figuré, métaphorique et indirecte de la langue en communication.

E. Largueche et C. Leguy (2011: 7-14) soulignent qu'il n'est pas toujours facile dire les choses de façon directe. Elles affirment qu'il existe des moyens qui permettent aux participants dans une conversation de contourner les difficultés et de rester dans les normes d'une communication socialement acceptée. La parole indirecte dont elles parlent peut être motivée par la nécessité et le désir d'éviter une situation trop délicate, un sujet interdit ou une certaine agressivité dans l'expression d'une pensée, d'un sentiment ou

${ }^{1}$ LADL : Laboratoire d'Analyse Documentaire et Linguistique - 1'Université Paris 7. LLI : Laboratoire de Linguistique Informatique - l'Université Paris 13. 
d'une attitude. Cette manière de communiquer serait codée par les normes de société.

C. Leguy cite l'exemple de l'emploi du proverbe dans un certain contexte qui, de façon indirecte, peut transmettre une critique à des participants de la conversation et qui est très souvent compréhensible uniquement à ses destinataires (2011: 157-174). Elle conclut que la manière indirecte d'adresser la parole peut s'expliquer d'une part par certaines circonstances et le contexte, et d'autre part, par les conventions et les normes sociales.

Nous considérons que l'empoi interprétatif c'est-à-dire, non littéral de la langue constitue une des stratégies majeures de communication qui est censée s'acquérir et se développer de façon graduelle et c'est pourquoi elle nécessite être contenue dans l'apprentissage de français langue étrangère. L'un des pas nécessaires dans la réalisation d'un tel objectif serait l'introduction systématique des expressions idiomatiques dans les manuels de français.

C'est un code communicatif qui rend les apprenants capables de parole. Ce code est réalisé par l'intégration des expressions idiomatiques dans la leçon.

Par la suite, nous allons répondre à plusieurs questions qui ont pour l'objectif de faire un état des lieux dans la problématique concernant le sujet mentionné.

Les programmes scolaires visant le français langue étrangère au Monténégro et au Massachussets prévoient-ils l'apprentissage des expressions idiomatiques ?

Pour comprendre si les programmes de français langue étrangère au Monténégro et au Massachussets prévoient l'apprentissage des expressions idiomatiques, nous avons tenu indispensable de consulter le contenu de programmes de français.

$\mathrm{Au}$ Monténégro, le programme scolaire pour l'école élémentaire (Bogojevic et al., 2005), de la IVe à la IXe année (les élèves âgés de 8 à 14 ans), ensuite les programmes de français première langue étrangère pour le lycée (Djukanovic - Bogojevic et al., 2005), de la Ire à la IVe année ainsi que le programme de français seconde langue étrangère pour le lycée (Djukanovic - Bogojevic et al., 2005), de la Ire à la IVe année. Nous présenterons ci-dessous les résultats de cette recherche.

Tous les programmes consultés soulignent l'importance des composantes interculturelles et éthiques dans l'enseignement. Elles visent les objectifs éducatifs et l'acquisition des connaissances interculturelles. Les objectifs de l'enseignement de français sont entre autres éthiques, sociaux et émotionnels. Ce sont les objectifs suivants: favoriser l'esprit de la tolérance, accepter les codes de comportement, s'approprier de savoir-faire communicatifs dans un contexte multiculturel, prendre un rôle actif dans son milieu social, combatte les préjugés, développer la confiance en soi et une attitude positive envers soi et les autres.

Nous considérons que les objectifs généraux, les sujets (par exemple: relation familiale, actualité, société, et autres sujets), les contenus et les 
objectifs opérationnels des programmes de français présentent un cadre suffisamment vaste pour y introduire l'apprentissage des expressions idiomatiques. Nous nous en servons très souvent pour exprimer la façon d'être, les choses qui nous concernent personnellement, pour parler de nos relations avec les autres, pour décrire le monde qui nous entoure. Ces expressions parlent de l'homme en tant qu'être social. Elles peuvent être utilisées comme une façon indirecte pour décrire son entourage et les relations qui s'y instaurent. Les expressions idiomatiques assurent une sorte d'économie de mots puisqu'en très peu de mots elles nous permettent d'exprimer une signification complexe.

Le programme de français pour l'école élémentaire (2005: 13-50) assure ainsi un cadre nécessaire pour envisager l'apprentissage de moyens lexicaux servant à exprimer de façon indirecte les pensées, les émotions, les prises de positions, les attitudes. Pourtant, les savoir-faire présentés dans ces programmes ne prévoient pas l'apprentissage d'expressions au sens figuré, la compréhension de mots en contexte, ni la compréhension d'émotions ou attitudes qui ne sont pas explicitement exprimées. La conclusion qui nous paraît juste c'est l'intégration des expressions idiomatiques dans le programme. Elle s'avère le moyen incournable permettant non seulement d'exprimer et de parler d'émotions, d'attitudes, et meme de porter un jugement mais, aussi d'établir des relations au sein de la société.

En ce qui concerne le programme de français seconde langue étrangère au lycée (2005: 43-71) nous notons qu'il assure les conditions nécessaires et un cadre suffisamment vaste pour l'apprentissage systématique des expressions idiomatiques. Outre les objectifs, les sujets et les contenus, le programme met en exergue les compétences de la compréhension du sens de mots dans le contexte. L'apprenant comprend les attitudes et les émotions à l'aide du contexte. Les savoir-faire, les compétences et les fonctions communicatives prévues par le programme comprennent l'expression de sentiments, d'attitudes et la description de sa propre expérience. Pourtant, nous n'avons trouvé aucune mention concernant le sens figuré ni les expressions idiomatiques. En conclusion, nous trouvons qu'il est indispensable d'introduire de façon explicite les expressions idiomatiques ainsi que d'autres formes de l'emploi non littéral de la langue, d'autant plus que le cadre du programme de la seconde langue étrangère prévoit les objectifs, les sujets, les contenus, les compétences, les savoir-faire et les fonctions communicatives se référant à la vie émotionnelle et sociale de l'être humain.

De nos jours, de nombreuses études s'occupant des expressions idiomatiques témoignent de l'importance de ce segment du lexique français. Les résultats respectifs devraient être consultés dans le processus de la conception des programmes de langues, à l'égard de certains manuels de français dont nous parlerons dans le texte ci-dessous.

Outre cela, il faudrait souligner qu'au Monténégro le français est enseigné le plus souvent comme seconde langue étrangère le besoin d'enrichir le cadre de ce programmme est d'autant plus criant.

Le programme de français première langue étrangère pour le lycée (2005: 47-75) prévoit le travail sur le développement des compétences de 
compréhension des contenus implicites. Les savoir-faire prévus sont: reconnaître les les attitudes, les émotions, les circonstances de l'interaction, reconnaître le registre de la langue et les contenus implicites dans le texte. À différence des deux programmes mentionnés ci-dessus, celui-ci contient des savoir-faire qui se réfèrent à des attitudes et à des émotions qui ne sont pas exprimées de manière explicite. Dans les savoir-faire à l'écrit, le programme cite pour la première fois le mot «les expressions idiomatiques » (2005: 71). L'apprenant doit donc avoir le savoir-faire d'utiliser le vocabulaire approprié, les expressions idiomatiques.

Nous citons quelques fonctions communicatives prévues par ce programme: expressions des émotions, des modalités, des attitudes, des pensées et des convictions. Le programme prévoit ainsi l'apprentissage de formes lexicales complexes et les expressions au sens figuré. Tout de même, vu que le programme contient différents genres (essai, critique littéraire, textes littéraires et poétiques), nous sommes d'avis qu'il faudrait y souligner davantage l'importance de la compréhension et de l'emploi de la parole indirecte, des tournures métaphoriques dans l'expression des émotions et des attitudes, puisque ce sont des éléments importants à travers lesquels la langue française véhicule les contenus implicites socioculturels. Dans ce cadre, les expressions idiomatiques devraient avoir une place importante.

Or, le français n'est pas étudié majoritairement comme première langue étrangère au Monténégro. C'est pourquoi nous considérons que la partie de son contenu se référant aux expressions idiomatiques devrait également trouver sa place dans le programme de français seconde langue étrangère afin d'y remplir des lacunes liées à l'emploi non littéral de la langue.

Heureusement, ce problème trouve parfois sa solution dans les situations pratiques en classe de langue puisque certains manuels de français seconde langue étrangère prévoient les contenus visant certaines expressions idiomatiques et des tournures métaphoriques.

$\mathrm{Au}$ Massachussetts, nous avons observé un cadre obligatoire qui comprend cinq composantes du programme scolaire langue étrangère (Massachusetts Foreign Languages Curriculum Framework, August 1999). Il s'agit là d'un outil communément interpelé « les $5 \mathrm{c} »{ }^{1}$ qui permet d'intégrer les expressions idiomatiques dans la didactique des langues étrangères.

Malgré le fait que les expressions idiomatiques ne sont pas citées dans les programmes ni mentionnées de manière systématique dans les manuels, nous avons constaté qu'il y a un nombre de professeurs de français conscients de l'importance de ce segment de lexique. Ils trouvent nécessaire d'incorporer une expression idiomatique dans chaque unité.

Nous allons présenter ci-dessous les résultats de l'analyse du contenu de plusieurs méthodes de français concernant la présence des expressions idiomatiques.

${ }^{1}$ Les composantes de ce curriculum est au nombre cinq : Communication, Cultures, Comparaisons, Connections, et Communautés (Communication, Cultures, Comparisons, Connections, and Communities). 


\section{Quelle est la place des expressions idiomatiques dans les manuels de français?}

Afin de répondre à la question concernant la place des expressions idiomatiques dans les manuels de français, nous avons analysé les manuels utilisés au Monténégro mais aussi dans d'autres pays puisque ces manuels sont publiés par les maisons d'édition françaises. Les manuels de français suivants ont fait l'objet de notre recherche: Belleville 2 (Gallier - GrandeClement, 2004) et Belleville 3 (Gallier - Grande-Clement, 2005), Panorama 2 (Girardet - Cridlig, 2004), Junior plus 2 (Butzbach et al., 2006), Junior 4 (Butzbach et al., 2000), Campus 2 (Girardet - Pecheur, 2002), Campus 4 (Courtillon - Guyot-Clement, 2005), Echo Junior A2 (Girardet - Pecheur, 2012) et Echo Junior B1 (Girardet - Pecheur, 2012) publiés par la maison CLE International, ainsi que les manuels Reflets 1 (Capelle - Gidon, 1999), Alter Ego 1 (Berthet et al., 2006), Alter Ego + 3 - B1 (Dollez - Pons, 2007), Alter Ego 4 - B2 (Berthet, 2008), Alter ego 5 - C1/C2 (Berthet - Louvel, 2010), et Le français des relations européennes et internationales A1/A2 (Riehl et al., 2011), publiés par la maison Hachette.

L'analyse du contenu des manuels mentionnés ci-dessus a montré que les expressions idiomatiques n'y sont pas traitées de la même façon. Dans certains d'entre eux, nous observons l'absence presque totale d'expressions idiomatiques tandis que dans certains autres elles y sont présentes dans les textes ou dialogues qui peuvent être accompagnés de brèves explications. Cependant, il y a un nombre restreint de manuels qui accordent une attention considérable à l'apprentissage des expressions idiomatiques et qui y consacrent une leçon entière sur une ou deux pages à l'intérieur d'une unité. Dans la conception de méthodes de français il existe une certaine conscience sur l'importance de ce segment du lexique français pour l'apprentissage de français langue étrangère malgré les inconsistances observées d'un manuel à l'autre.

Les expressions idiomatiques ne figurent pas dans les manuels destinés à l'école élémentaire au Monténégro : Junior plus 1 (Butzbach et al., 2006), Junior plus 2 (Butzbach et al., 2006), Junior plus 3 (Butzbach et al., 2006). Cela correspond à la même lacune dans les programmes de français pour ce niveau.

En ce qui concerne le lycée, le manuel Junior 4 (Butzbach et al., 2000), qui avait été utilisé il y a quelques années, consacre une page entière dans chaque unité à des expressions imagées. Ces expressions y sont accompagnées d'amusants dessins comiques qui traduisent le sens littéral et inapproprié, tandis que leur véritable sens, évidemment figuré, est expliqué à l'aide de courts exercices de compréhension.

Le manuel Reflets 1 (Capelle - Gidon, 1999) réserve également une page entière aux expressions idiomatiques (Des mots pour le dire) qui contiennent différents types de constituantes et se réfèrent à des sujets variés (par exemple: la santé etc.). L'organisation de cette page est conçue comme dans le cas précédent: un dessin qui caricature l'expression en démontrant que la vraie signification de l'expression n'est pas littérale. Cette même page contient les exercices respectifs destinés à la compréhension des expressions 
(les exercices d'association) et à la recherche des équivalents en langue maternelle des apprenants.

Le manuel Panorama 2 (Girardet - Cridlig, 2004) qui avait été utilisé auparavant dans l'enseignement de français n'a pas de leçons consacrées aux expressions idiomatiques, mais il est possible de repérer ces dernières dans les textes et les exercices respectifs dont l'objectif vise l'apprentissage du vocabulaire.

Les méthodes de français Campus 2 (Girardet - Pecheur, 2002) et Campus 4 (Courtillon - Guyot-Clement, 2005) ne prévoient non plus de leçons particulièrement destinées à l'apprentissage des expressions idiomatiques. Il est tout de même possible de remarquer quelques expressions dans les dialogues et des exercices dont l'objectif est l'apprentissage du vocabulaire.

Dans les manuels Alter Ego 1 (Berthet et al., 2006) et Alter Ego 3 (Dollez - Pons, 2007) nous n'avons pas observé de leçons ni d'exercices qui traitent des expressions idiomatiques.

Par contre, les manuels Alter Ego B2 (Berthet, 2008) et Alter Ego C1/C2 (Berthet - Louvel, 2010) contiennent des expressions ayant le sens figuré. Elles figurent dans la partie Lexique. Les activités prévues dans les exercices visent la sensibilisation des apprenants au sens littéral et au sens figuré des mots et des expressions. Cependant, nous n'avons pas pu constater un effort suffisant pour introduire les expressions idiomatiques de façon systématique.

Belleville 2 (Gallier - Grande-Clement, 2004) et Belleville 3 (Gallier - Grande-Clement, 2005), manuels actuellement en usage dans les écoles secondaires au Monténégro, ont introduit ce segment de lexique dans leur programme, plus précisément dans les parties textuelles. Les expressions idiomatiques sont ensuite présentées et traitées dans les exercices destinés à la compréhension du lexique. Les pages prévues pour la révision et l'évaluation contiennent également les expressions imagées. En ce qui concerne le choix, nous avons noté la présence d'expressions contenant les composantes désignant le corps humain, mais le choix d'expressions peut également être motivé par des thèmes comme: l'argent, la situation économique, etc. En plus, le manuel contient les textes poétiques ( $C$ 'était un bon copain, R. Desnos) très intéressants contenant les expressions idiomatiques ce qui rend leur apprentissage simple et amusant.

À partir de septembre 2015, les manuels Echo Junior A2 (Girardet Pecheur, 2012) et Echo Junior B1 (Girardet - Pecheur, 2012) seront utilisés dans l'enseignement de français langue étrangère dans les écoles secondaires. Après avoir consulté et analysé le contenu et la conception de cette méthode, nous sommes en mesure d'observer que seule la leçon zéro du manuel Echo Junior B1 (Girardet - Pecheur, 2012) dans la partie qui prépare l'apprenant à l'utilisation du livre et à des techniques d'apprentissage fait référence à la compréhension de mots et d'expressions en contexte. L'une des stratégies d'apprentissage de français langue étrangère suggérées par quelques auteurs est l'utilisation d'un dictionnaire monolingue. Ils citent l'exemple d'une définition lexicographique et les activités à réaliser à partir de cette définition. En voici quelques exemples : chercher dans la définition des synonymes, 
l'origine de mots, le sens figuré. Pourtant, après ce premier conseil, les leçons et les unités de ces deux manuels ne prévoient pas le travail sur les expressions idiomatiques. Il est vrai qu'il existe un nombre restreint d'expressions avec les constituantes qui désignent les couleurs, mais il n'y a pas de leçons, pages ou exercices particulièrement conçus pour expliquer les expressions idiomatiques qui pourraient se trouver dans les textes et dialogues. À la différence des méthodes Belleville 2 (Gallier - GrandeClement, 2004) et Belleville 3 (Gallier - Grande-Clement, 2005), les méthodes de français qui seront en usage dans les écoles secondaires au Monténégro à partir de septembre 2015 ne contiennent pas de contenus qui se réfèrent à l'apprentissage systématique des expressions idiomatiques, nous constatons.

Nous constatons l'inconsistance de la part des auteurs vis-à-vis ce segment du lexique français. Ce fait est d'autant plus évident si l'on prend en considération le fait que les méthodes Belleville et Echo Junior sont publiées par la même maison d'édition, CLE International. Nous pouvons conclure que les auteurs n'accordent pas la même importance aux expressions idiomatiques dans l'apprentissage de français langue étrangère.

En nous référant particulièrement au programme scolaire de l'état de Massachusetts, nous avons constaté une façon de résoudre ce problème. Bien que la mention de l'emploi des expressions idiomatiques n'est pas systématiquement prévue dans les manuels, le fait que chaque unité prévoit trois compétences didactiques, linguistique, culturelle et communicative, et vu que les enseignants sont obligés de créer leurs leçons autour de « $5 \mathrm{c}$ », les expressions idiomatiques y trouvent leur place. Dans notre recherche nous avons pu trouver plusieurs de ces professeurs qui volontairement choisissaient les expressions idiomatiques appropriées à la leçon du jour ou à l'unité qui va être explorée.

L'étude de ces expressions souvent prend forme de dictée, de discusssions en contexte ou encore, d'étude étymologique de l'expression. Une telle stratégie permet à l'apprenant d'être capable de parole. Prenons l'exemple suivant: l'expression Petit à petit l'oiseau fait son nid, peut être utilisée comme un code qui sert de prétexte pour travailler sur la prosodie, la dictée, la liaison, la polysémie de certains éléments constitutifs de l'expression, etc.

\section{Quelle stratégie adopter afin d'assurer aux expressions idiomatiques une place importante dans la classe de français langue étrangère?}

La réponse à la question posée ci-dessus est complexe. Elle exige la mobilisation de plusieurs sujets et la réalisation de plusieurs activités. Tout d'abord, nous considérons qu'il est nécessaire d'élargir les programmes de français de sorte que les expressions idiomatiques y trouvent leur place. Puis, à notre avis, il faudrait organiser une ou plusieurs journées d'étude consacrées à des discussions sur la stratégie dans l'enseignement de français et sur l'importance et la place des expressions et locutions idiomatiques dans les programmes d'enseignement de français. Outre cela, il faudrait publier des travaux de recherche en didactique visant l'exploitation des expressions 
idiomatiques en classe. Ces articles auraient pour l'objectif de sensibiliser les enseignants à cette problématique et les motiver à donner leur contribution à la popularisation de ce sujet. Ils devraient être publiés dans les revues ayant une distribution régulière dans les établissements scolaires. Nous sommes donc d'avis que ce contenu devrait être plus présent dans les méthodes de français. Ensuite, nous trouvons indispensable à ce que les librairies et les bibliothèques offrent aux lecteurs un choix plus vaste de dictionnaires de locutions et d'expressions monolingues et bilingues. Nous aimerions souligner encore une fois qu'il faudrait accorder une place obligatoire aux expressions idiomatiques dans les programmes de français.

\section{Les résultats d'une recherche visant les expressions idiomatiques et leur application en didactique}

La conclusion que nous venons d'exposer ci-dessus implique logiquement d'autres idées et pose un nouveau défi qui concerne la nécessité de concevoir des techniques de classe dont l'objectif serait de proposer comment enseigner les expressions idiomatiques. La réalisation de cette idée permettrait d'appliquer des résultats provenant de recherche visant cette partie du lexique français.

Dans notre cas, ce seraient les résultats obtenus lors de la réalisation de notre thèse de doctorat qui traite des caractéristiques sémantiques des expressions idiomatiques. $\dot{A}$ ce propos, nous allons citer une partie de résultats qui pourraient être appliqués dans la didactique de français langue étrangère. Ils relèvent des aspects lexicaux, syntaxiques, sémantiques, lexicographiques et socioculturels de l'emploi des expressions idiomatiques dont l'une des constituantes désigne le corps humain.

Nous sommes persuadés que les définitions lexicographiques des expressions idiomatiques que nous avons explorées constituent une source importante pour le travail sur le lexique et le vocabulaire. Ce sont des périphrases définitionnelles qui contiennent des synonymes et des exemples d'emploi des expressions dans différents contextes et registres de langue. Ensuite, les résultats de l'analyse sémique à partir de leurs définitions lexicographiques fournissent un riche répertoire lexical lié à ces expressions qui comprend toutes les classes et catégories grammaticales susceptibles de devenir un moyen efficace lors de l'enseignement et l'apprentissage du lexique, de la grammaire et lors de la création d'exercices. Nous avons pu voir dans le texte ci-dessus que certains manuels suggèrent le travail sur la définition lexicographique (Girardet - Pecheur, Echo Junior B1, 2012). De plus, de nombreux linguistes soulignent leur importance dans les recherches en ce domaine ce qui nous motive davantage de baser nos techniques didactiques sur le contenu des définitions lexicographiques. A. J. Greimas avait envisagé ce procédé dans sa conception d'analyses sémantique et sémique. Il trouve que le caractère le plus évident de la définition lexicographique, en regard des analyses sémantiques, c'est son aspect du discours ordinaire, ceci par destination, puisqu'elle doit être comprise de n'importe qui (francophones dans le cas particulier) (1966a : 46). De son côté, Josette Rey-Debove dans son Étude linguistique et sémiotique des dictionnaires français contemporains, souligne que la définition 
lexicographique doit être considérée d'abord comme une périphrase synonymique, mais aussi comme une analyse du contenu. Elle évoque d'ailleurs souvent dans son ouvrage que les sémanticiens s'en servent pour analyser le contenu (1971: 195).

Nous aimerions mettre en avant un autre point important que nous avons relevé justement grâce à ce travail d'analyse des périphrases définitionnelles. Il concerne l'interprétation de l'emploi non littéral de la langue c'est-à-dire des idées que certaines expressions véhiculent et qui élargissent la compréhension des éléments sémantiques et socioculturels qui y sont contenus. Se rendre compte du lien entre l'expression idiomatique et un contexte précis qui concerne l'existence humaine, la vie de tous les jours de l'être humain et ses activités, peut sensibiliser les apprenants à l'emploi de cette partie du lexique français. Cela rend l'apprentissage de langue plus subjectif et par conséquent plus efficace et plus simple.

À titre d'exemple, nous citerons ci-dessous quatre expressions idiomatiques dont l'une des constituantes est le lexème: «la dent». Nous mettrons à la disposition de nos collègues enseignants et aux auteurs de méthodes de français une partie de périphrases définitionnelles se référant aux expressions respectives ainsi qu'une partie de résultats obtenus lors de l'analyse sémique de ces périphrases. Les expressions idiomatiques seront suivies des caractéristiques sémantiques et contextuelles issues de l'analyse citée précédemment. Cela ne serait qu'une invitation à la réflexion sur les perspectives de travail de classe à explorer.

Ci-dessous nous présenterons tout d'abord deux expressions idiomatiques dont la caractéristique la plus évidente est de correspondre à des messages fonctionnels (Greimas, 1986) désignant un procès. Ensuite, nous présenterons deux autres expressions idiomatiques en tant que messages qualificatifs (Greimas, 1986) désignant une qualification ou un état. Les quatre expressions auront pour lexème central : «la dent ».

\section{Messages fonctionnels :}

1. "Se faire les dents" = fig. s'entraîner, s'aguerrir est un message fonctionnel désignant le procès.

Voici un extrait du contenu de la périphrase définitionnelle de l'expression idiomatique citée (Le Grand Robert de la langue française, version électronique) :

S'entraîner v. pronominal : Faire l'apprentissage de, en s'habituant.

1. V. tr. Préparer (un animal, une personne, une équipe) à des performances, au moyen d'exercices appropriés.Exerce. Par ext. Entraîner des soldats. Aguerrir, dresser, endurcir, former. — Pron. S'entraîner au maniement des armes.

s'aguerrir: 2. Fig. Accoutumer (qqn) aux choses pénibles. Affermir, cuirasser, endurcir, tremper

Endurcir v. tr.: 2. (Compl. humain). Rendre moins sensible physiquement; rendre plus dur au mal, rendre résistant.

3. Rendre moins sensible moralement.Bronzer (3.), cuirasser, dessécher, durcir. 
s'endurcir v. pron.: Devenir plus résistant (en parlant du corps, de la peau, des muscles...). (1636). Devenir plus endurant, plus robuste, plus vigoureux; s'accoutumer à la fatigue, à la douleur, aux privations. 3. (1647). Devenir moins sensible, plus dur.

CONTR. Amollir, attendrir, émouvoir, fléchir, ramollir, toucher. - (Du p. p.) Amolli, aveuli, contrit, efféminé, ramolli.

Les caractéristiques sémantiques générales de cette expression sont :

$$
\begin{gathered}
\text { sens figuré vs sens propre } \\
\text { animé vs inanimé } \\
\text { humain vs animal } \\
\text { activité vs passivité } \\
\text { fonction vs qualification } \\
\text { dynamisme vs statisme } \\
\text { ou action vs inertie, }
\end{gathered}
$$

tandis que ses caractéristiques spécifiques seraient les suivantes :

absent vs présent

conscient vs inconscient

créant vs créé

transformant vs transformé

formant vs formé

combattant vs calme.

Les contextes désignant les sphères de la vie réelle concernant cette expression sont : comportement moral et psychique, sentiments.

Cette expression idiomatique parle de l'être humain et elle le fait d'une manière métaphorique. Au niveau sémantique elle parle de la nature humaine. Le signifiant est complexe, doté de double image et il referme un signifié métaphorique. Nous avons conçu plusieurs procédés d'analyse qui nous ont permis de décomposer en profondeur le contenu de ces messages. Nous sommes d'avis que le contexte « comportement moral et psychique » se réfère à des émotions, des humeurs, des réflexions, des attitudes morales, des aptitudes morales et des actions qui en découlent par exemple : « susciter des émotions chez soi ou chez l'autre », « exprimer des émotions ». Ces contextes montrent que les expressions idiomatiques jouent un rôle important dans l'existence de l'être humain et dans sa communication avec les autres. Elles l'aident à exprimer sa personnalité, ses émotions et humeurs, à créer des liens émotifs et intellectuels avec les autres êtres humains au sein de la société. Il communique sa personnalité et ses attitudes intellectuelles et morales indirectement, à travers la métaphore.

2. "Donner un coup de dent" = métaphoriquement: "attaque, critique acerbe " est un message fonctionnel désignant un procès.

Voici un extrait du contenu de la périphrase définitionnelle de l'expression idiomatique citée (Le Grand Robert de la langue française, version électronique) : Attaque n. f. : Fig. 1. (1596). Généralement au plur. Paroles ou écrits qui critiquent durement - Accusation, coup. 
Critique n. f. : C. Cour. Action de critiquer, tendance de l'esprit à émettre des jugements sévères, défavorables, négatifs (la critique); jugement défavorable (une, des critiques). Attaque, blâme, censure, condamnation, contradiction, glose; critiquer.

Acerbe adj. : Didact. Aigre et âpre. 2. Fig. et cour. Qui cherche à blesser; qui critique avec méchanceté. Une personne acerbe. Acrimonieux, agressif, blessant, caustique, sarcastique.

Les caractéristiques sémantiques générales de cette expression sont :

sens figuré vs sens propre

animé vs inanimé

humain vs animal

activité vs passivité

fonction vs qualification

dynamisme vs statisme ou action vs inertie

manière d'agir vs manière d'être,

tandis que ses caractéristiques spécifiques seraient les suivantes :

antipathique vs sympathique

négatif vs positif

blessant vs blessé

jugeant vs tolérant

suscitant, provoquant émotions vs éprouvant, exprimant émotions.

Les contextes désignant les sphères de la vie réelle concernant cette expression sont: comportement, relations humaines, émotions, communications de messages, attitudes.

Le contexte « relations » est un contexte global qui referme toute l'existence de l'homme en tant qu'être social. L'homme agit et réagit dans la communication avec les autres. Ce sont ces E.I. à l'aide desquelles l'homme communique ses pensées, idées, émotions et humeurs. C'est sa manière de communiquer, faire passer le message.

\section{Messages qualificatifs :}

1. "Avoir, garder une dent contre quelqu'un" = "lui garder rancune ", " être passionnée par quelque chose " est un message qualificatif désignant un état ou la description.

Voici un extrait du contenu de la périphrase définitionnelle de l'expression idiomatique citée ci-dessus (Le Grand Robert de la langue française, version électronique) : Rancune n. f.: Souvenir vif et tenace que l'on garde d'une offense, d'un mal, avec de l'hostilité et un désir de vengeance* (la rancune); disposition ou état d'âme de celui qui garde un tel ressentiment (une, des rancunes). Aigreur, animosité (cit. 8), rancœur, ressentiment, haine, hostilité, malveillance.

Ressentiment n. m.: 2. Le fait d'éprouver encore, de se souvenir avec animosité des maux, des torts qu'on a subis. $\square$ Rancœur, rancune. Animosité n. f. : Sentiment persistant de malveillance qui porte à nuire à qqn. 2. Vieilli. Emportement, violence (dans une discussion, un affrontement, un combat...). 
Acharnement, âpreté, ardeur, chaleur, colère, emportement, véhémence, violence. CONTR. Amour, amitié, bienveillance, cordialité, sympathie. Agressivité n. f. : 1. Caractère agressif. Combativité, malveillance, violence. CONTR. Douceur, bienveillance, humilité, modération.

Les caractéristiques sémantiques générales de cette expression sont :

$$
\begin{gathered}
\text { sens figuré vs sens propre } \\
\text { animé vs inanimé } \\
\text { humain vs animal } \\
\text { qualification vs fonction } \\
\text { manière d'être vs manière d'agir, }
\end{gathered}
$$

tandis que ses caractéristiques spécifiques seraient les suivantes :

$$
\begin{gathered}
\text { combatif vs calme, indulgent } \\
\text { (sent.) négatif vs positif } \\
\text { passionné vs indifférent } \\
\text { insistant vs renonçant } \\
\text { persistant, durable vs passager } \\
\text { gardant vs négligeant } \\
\text { ressenti vs distant. }
\end{gathered}
$$

Les contextes désignant les sphères de la vie réelle concernant cette expression sont : état, sentiments, attitude morale, relations humaines.

2. "Avoir la dent " fam." = "avoir faim " est un message qualificatif désignant un état ou la description.

Voici un extrait du contenu de la périphrase définitionnelle de l'expression idiomatique citée (Le Grand Robert de la langue française, version électronique) : Faim n. f. : 1. Sensation qui, normalement, accompagne le besoin de manger. 2. Fig. Appétit, besoin éprouvé. Faim intellectuelle. Appétit, besoin, désir, envie, soif. Faim de gloire, d'honneurs (Ambition, cit. 1), de richesses (Avidité, cupidité). Manger v. tr. : 1. a. Avaler pour se nourrir (un aliment solide ou consistant) après avoir mâché. Absorber, avaler, consommer, dévorer, Dévorer (un être vivant, une proie). (1694). Fig. Manger quelqu'un des yeux, le regarder avidement, avec admiration, amour, convoitise, désir...Dévorer.

Les caractéristiques sémantiques générales de cette expression sont :

$$
\begin{aligned}
& \text { sens figuré vs sens propre } \\
& \text { animé vs inanimé } \\
& \text { humain vs animal } \\
& \text { qualification vs fonction } \\
& \text { manière d'être vs manière d'agir, }
\end{aligned}
$$

tandis que ses caractéristiques spécifiques seraient les suivantes : 
Les contextes désignant les sphères de la vie réelle concernant cette expression sont : sensation et besoins physiques.

Le contexte "état» se réfère aussi à l'être humain, à son appréhension du monde et de sa propre existence. C'est sa manière d'être pour soi, un monde intérieur qui existe en tant que tel. Il parle de l'impact qu'exerce l'intérieur, l'intimité, tout ce qui est essentiel pour un être humain, sur la communication. Un grand nombre d'expressions idiomatiques en effet ont été créées pour décrire métaphoriquement l'être humain et son monde intérieur et intime qu'est le monde des états d'âme, de la manière d'être, des émotions, humeurs, attitudes et aptitudes. C'est ce qui rend chaque être humain un individu original et unique.

Comme nous l'avons indiqué plus haut, le contexte «relations » est un contexte global qui referme toute l'existence de l'homme en tant qu'être social. L'homme agit et réagit dans la communication avec les autres. Ce sont ces E.I. à l'aide desquelles l'homme communique ses pensées, idées, émotions et humeurs. C'est sa manière de communiquer, de faire passer le message. Nous constatons qu'une expression idiomatique peut se référer à plusieurs contextes. Il en résulte que les expressions idiomatiques représentent un moyen lexical qui reflète l'homme en tant qu'être émotif et social. L'homme se sert d'expressions idiomatiques pour parler de façon subjective de soi, de ses émotions, de ses relations avec les autres. Les expressions idiomatiques observées sont toujours empreintes d'une émotion, elles expriment une attitude et le désir de dire plus, mais de façon imagée et métaphorique. Cette conclusion évoque, une fois de plus, la thèse de G. Lakoff et M. Johnson (1980) d'après laquelle la métaphore fait partie de la vie de tous les jours et qu'à travers la métaphore l'homme exprime soi-même et le monde qui l'entoure.

Ci-dessus nous avons présenté une base de données sémantique et lexicale à partir de laquelle il serait possible de créer des activités dont l'objectif serait l'apprentissage de français et de travail en classe de langue. Ce contenu pourrait être mis à la disposition pour un éventuel livre de professeur. Les activités que nous avons envisagées dépendent du niveau de l'apprenant et peuvent être les suivantes :

a. le jeu d'association d'idées à partir d'une expression idiomatique,

b. l'activité de reformulation d'idées,

c. le travail sur les synonymes et les antonymes,

d. le travail avec le dictionnaire,

e. l'analyse de registres de langues et de différents styles fonctionnels,

f. le travail en petits groupes sur les dialogues (l'emploi de l'expression et de sa périphrase selon le registre et le contexte),

g. l'écriture créative (rédaction de textes avec une ou plusieurs expressions données ; concours de meilleur essai),

h. le travail créatif (concours de meilleure caricature, affiche, bande dessinée),

i. la réflexion sur la composition de nouvelles expressions idiomatiques, 
j. l'analyse syntaxique et morphosyntaxique des expressions idiomatiques.

En conclusion, nous espérons que certaines questions soulevées dans cet article attireront l'attention des enseignants de français ainsi que des responsables de la politique didactique de français et des maisons d'édition.

\section{Bibliography}

BERTHET, A. et al. 2006. Alter Ego1, Methode de français. Hachette. ISBN 9782011554208.

BERTHET, A. 2008. Alter Ego 4 - B2, Cahier d'activites. Hachette. ISBN 13: 978-2011555175.

BERTHET, A. - LOUVEL, C. 2010. Alter Ego5 - C1/C2, cahier de perfectionnement. Hachette. ISBN 13: 9782011557988.

BOGOJEVIC, D. et al. 2005. Predmetni programi : francuski jezik, IV, V, VI, VII, VIII i IX razred devetogodišnje osnovne skole : ruski jezik, IV, V, VI, VII, VIII i IX razred devetogodisnje osnovne skole : italijanski jezik, IV, V, VI, VII, VIII i IX razred devetogodisnje osnovne skole : njemacki jezik, IV, V, VI, VII, VIII i IX razred devetogodisnje osnovne skole : spanski jezik, IV, V, VI, VII, VIII i IX razred devetogodisnje osnovne skole. Podgorica: Zavod za skolstvo, 2005. ISBN 86-85553-00-8.

BUTZBACH, M. et al. 2000. Junior 4, Methode de français. CLE International. ISBN 978-2-09-032360-3.

BUTZBACH, M. et al. 2006. Junior plus 1, livre de l'eleve. CLE International. ISBN 978-2-09-035400-3.

BUTZBACH, M. et al. 2006. Junior plus 2, Methode de français. CLE International. ISBN 978-2-09-032427-3.

BUTZBACH, M. et al. 2006. Junior plus 3, Methode de français. CLE International. ISBN 978-2-09-035408-9.

CAPELLE, G. - Gidon, N. 1999. Reflets 1, Methode de français. Hachette. ISBN 13: 978-2011551160.

COURTILlON, J. - GUIYOT-CLEMENT, C. 2005. Campus 4. CLE International. ISBN: 209-033314-6.

Dictionnaire des locutions françaises. 1957. sous la dir. de M. RAT, Librairie Larousse, Paris. ISBN 10: 2035349222.

Dictionnaire d'expressions et locutions. 2007. sous la dir. de A. REY et S. CHANTREAU, Ed. Dictionnaires Le Robert. Collection les Usuels, Paris. ISBN-13: 978-2849022665, ISBN-10: 2849022667.

DOLLEZ, C. - PONS, C. 2007. Alter Ego +3 B1, Methode de français. Hachette. ISBN 9782011555120.

DJUKANOVIC, B. - BOGOJEVIĆ. D. et al.2005. Nastavni predmet: drugi strani jezik : predmetni programi : engleski jezik I, II, III i IV razred opste gimnazije : francuski jezik I, II, III i IV razred opste gimnazije : ruski jezik I, II, III i IV razred opste gimnazije : italijanski jezik I, II, III i IV razred opste gimnazije : njemacki jezik I, II, III i IV razred opste gimnazije. Podgorica: Zavod za skolstvo, 2005. ISBN 86-85553-10-5.

DJUKANOVIC, B. - BOGOJEVIC. D. et al. 2005. Nastavni predmet : prvi strani jezik : predmetni programi : engleski jezik I, II, III i IV razred opste gimnazije : francuski jezik I, II, III i IV razred opste gimnazije : ruski jezik I, 